Nouveaux cahiers de la recherche en éducation

\title{
Apprendre et former : pour quelles réussites scolaires ?
}

\section{Olivier Dezutter et Colette Deaudelin}

Volume 10, numéro 1, 2007

Apprendre et former : pour quelles réussites scolaires?

URI : https://id.erudit.org/iderudit/1016854ar

DOI : https://doi.org/10.7202/1016854ar

Aller au sommaire du numéro

Éditeur(s)

Faculté d'éducation, Université de Sherbrooke

ISSN

1911-8805 (numérique)

Découvrir la revue

Citer ce document

Dezutter, O. \& Deaudelin, C. (2007). Apprendre et former : pour quelles réussites scolaires ? Nouveaux cahiers de la recherche en éducation, 10(1), 1-4. https://doi.org/10.7202/1016854ar d'utilisation que vous pouvez consulter en ligne.

https://apropos.erudit.org/fr/usagers/politique-dutilisation/ 


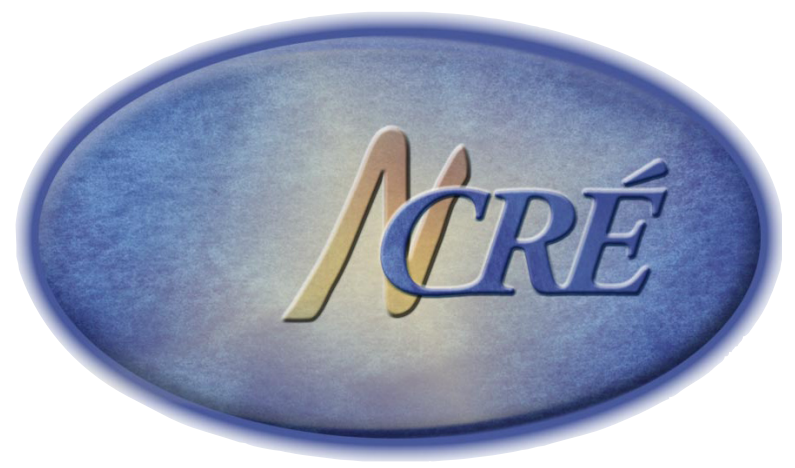

\title{
Introduction Apprendre et former: pour quelles réussites scolaires?
}

\author{
Olivier Dezutter et Colette Deaudelin \\ Université de Sherbrooke
}

La question de la réussite des jeunes est au cœur des préoccupations tant dans le domaine politique que dans ceux de la recherche et de la formation. Le ministère de l'Éducation du Québec, dans le cadre de l'implantation du renouveau pédagogique a réaffirmé le rôle essentiel de l'école à ce sujet, en lui assignant la triple mission d'instruire, de socialiser et de qualifier. En lien avec ce dernier aspect, les responsables du Ministère mettent l'institution scolaire et ses acteurs «en demeure d'assurer à tous la possibilité d'acquérir les moyens nécessaires pour mieux prendre leur place au travail, dans leur famille et dans la vie collective» (Gouvernement du Québec, 2001, p. 5). L'atteinte de tels objectifs apparaît difficilement mesurable mais plusieurs indices indiquent clairement que pour une frange non négligeable de la population scolaire, le parcours scolaire est semé d'embûches, loin d'aboutir aux résultats escomptés, marqué par l'échec et l'abandon.

Si, à l'échelle canadienne, le taux de décrochage - mesuré par le nombre d'élèves de 20 à 24 ans n'ayant pas de diplôme d'études secondaires et ne fréquentant pas l'école - tend à diminuer depuis 15 ans, il demeurait à près de 10\% en 2004-2005 (9,8\%). Ce taux est plus élevé dans certaines provinces dont le Manitoba, l'Alberta et le Québec, qui présentent respectivement des taux de $13,0 \%, 12,0 \%$ et $11,9 \%$ (Bowlby, 2005) ${ }^{1}$. Ce taux demeure particulièrement élevé dans les régions rurales et les petites localités dans ces mêmes provinces où il s'élève à $24,8 \%$, $21,0 \%$ et 19,4\% (Ibid.). Dans ces régions rurales, c'est donc un élève sur cinq qui n'a pas complété une formation du secondaire. Dans la région de l'Estrie, un jeune sur deux n'obtient pas son diplôme d'études secondaires à l'issue du cheminement normal de cinq ans.

1 Moyennes des années scolaires de 2002-2003 à 2004-2005. 
Cette situation ne prévaut pas uniquement au Québec et au Canada, comme en témoigne, pour la France, un récent rapport du Haut Conseil de l'Éducation dressant un lourd constat à propos de l'école primaire qui ne remplit pleinement son rôle, quant à l'acquisition du socle commun et à la préparation à la poursuite des études, que pour quelque $60 \%$ des élèves et ne parvient donc pas à «donner à tous les moyens adéquats et suffisants d'accéder à la réussite scolaire» (Haut conseil de l'Éducation, 2007, p. 8). Les auteurs allant jusqu'à prétendre que l'école primaire française semble s'être «résignée à l'échec des élèves qui accumulent les insuffisances» et «se révèle incapable de mettre en place un soutien et un rattrapage efficaces » (Ibid.).

Au-delà des constats chocs, des discours alarmistes et des polémiques qu'ils ne manquent pas de susciter, les initiatives se multiplient ces dernières années pour tenter de mieux comprendre le phénomène de l'échec scolaire dans sa pluridimensionnalité: personnelle, familiale et scolaire (Fortin, Marcotte, Royer et Potvin, 2006) en examinant autant les déterminants que les effets du binôme réussite/échec.

Malgré l'ampleur et la qualité des travaux réalisés jusqu'à présent sur cette thématique, les taux de décrochage conduisant à l'échec scolaire mentionnés précédemment demeurent inquiétants. Des pistes d'explications peuvent être formulées: une définition trop restrictive de la réussite scolaire, un regard scientifique qui est demeuré trop disciplinaire (psychologie, sociologie, etc.) alors que, par sa dimension systémique, cela exige un regard plus large, voire multidisciplinaire et, enfin, des lacunes importantes au plan du transfert des connaissances produites par la recherche vers les milieux de la pratique.

C'est dans ce contexte que la Faculté d'éducation de l'Université de Sherbrooke, insérée dans un milieu particulièrement sensible à la nécessité d'une mobilisation large en vue de contrer le décrochage scolaire ${ }^{2}$, a voulu contribuer à l'avancée de la réflexion en organisant les 11 et 12 octobre 2007, dans le cadre des dixièmes rencontres du Réseau international de recherche en éducation et en formation (RÉF), un colloque centré sur la thématique des réussites scolaires.

L'ambition du colloque était à la fois de mettre en question le concept de «réussite scolaire», de tenter de mieux comprendre la situation des jeunes et des adultes qui éprouvent de la difficulté à réussir dans l'école d'aujourd'hui, et d'interroger les pratiques des différents professionnels de l'éducation et des parents susceptibles de soutenir au mieux les élèves sur les voies de la réussite.

Le colloque a fait appel aux chercheurs de divers champs des sciences humaines et sociales (pédagogie, didactique, sociologie, psychologie, etc.) qui contribuent à l'étude des questions éducatives et de différents domaines de recherche en éducation. Trois axes de réflexion ont été privilégiés : les élèves et leurs difficultés, les interventions en classe et à l'école et les collaborations école et milieu.

Le présent dossier fait écho aux travaux présentés lors du colloque. Il regroupe quatre textes écrits au départ de communications présentées dans les différents axes précités. Les contributions émanent de chercheurs qui s'intéressent à des publics scolaires d'âges différents

2 Un plan régional de mobilisation et d'action a été décrété pour la période 2006-2016 par la Table estrienne de concertation interordres en éducation, qui regroupe les décideurs des commissions scolaires, des collèges, des universités et du ministère de l’Éducation, du Loisir et du Sport. 
et interrogent sous des angles particuliers la question des réussites scolaires en lien avec leur domaine de recherche: 1'apprentissage des mathématiques chez les adolescents et de jeunes adultes (Roditi), l'analyse des pratiques d'évaluation au service des apprentissages mises en œuvre par des enseignants du primaire en contexte de renouveau pédagogique (Deaudelin, Desjardins, Dezutter, Thomas, Corriveau, Lavoie, Bousadra et Hébert), l'analyse de l'évolution des difficultés de comportement et d'adaptation des jeunes fréquentant l'école primaire, qu'ils aient bénéficié de services éducatifs spécialisés et individualisés (Déry, Laventure, Toupin, Verlaan et Pauzé) ou qu'ils aient été rassemblés dans un espace spécialement conçu pour eux, baptisé la classe «Kangourou» (Couture et Lapalme).

Le degré de maîtrise de la langue maternelle et des mathématiques est un facteur reconnu parmi les facteurs de protection au regard du décrochage scolaire. Éric Roditi, spécialiste de la didactique des mathématiques, cherche à mieux comprendre l'activité mathématique vécue par les apprenants afin de mieux comprendre et prévenir les difficultés d'apprentissage. Dans l'article «Aider les élèves à apprendre à comparer des nombres décimaux», il rend compte des résultats d'une recherche quantitative portant sur la comparaison des nombres décimaux, menée auprès de plus de 400 élèves et étudiants français de 10 à 25 ans. Il examine entre autres l'influence sur la performance des personnes testées de la forme de présentation des nombres ou encore de la présence ou non d'un contexte. Soucieux de mettre à l'épreuve de la pratique les éléments résultant de la vaste enquête effectuée, Roditi fait ensuite écho à l'expérimentation d'un scénario d'aide mis en œuvre par une enseignante volontaire avec un groupe d'élèves du début du secondaire. L'étude démontre l'efficacité de la démarche consistant à amener les élèves en difficulté à mettre en relation plusieurs traitements des nombres dans différentes situations et à confronter leurs raisonnements corrects ou erronés qui justifient ces traitements.

L'article de Deaudelin, Desjardins, Dezutter, Thomas, Corriveau, Lavoie, Boussadra et Hébert fait état d'une étude visant la diffusion de pratiques exemplaires témoignant de l'appropriation de changements prescrits par le renouveau pédagogique québécois relativement à l'évaluation des apprentissages. Les pratiques d'évaluation formative de 13 enseignants du primaire y sont analysées à travers 25 activités d'apprentissage-enseignement mises en œuvre dans différentes disciplines. L'analyse a porté sur les dimensions comportementale et cognitive des pratiques en examinant plus spécifiquement les processus sollicités, les dispositifs mis en œuvre ainsi que les contenus traités. Les résultats montrent que les participantes et participants ont intégré à leurs pratiques plusieurs des caractéristiques de l'évaluation formative préconisée par le ministère de l'Éducation, et que leur principal défi consiste à partager la responsabilité des processus de régulation avec l'élève en créant des conditions pour que celui-ci développe sa capacité à autoréguler ses apprentissages.

Les deux autres articles émanent d'équipes de chercheurs spécialisés dans l'étude des inadaptations sociales de l'enfance. Ils relèvent tous deux d'une démarche évaluative portant sur des pratiques d'accompagnement d'élèves québécois du primaire, diagnostiqués précocement en difficulté de comportement, et dont le nombre a constamment augmenté depuis les quinze dernières années.

Déry, Laventure, Toupin, Verlaan et Pauzé cherchent à évaluer les effets dans le temps des services offerts aux élèves du primaire chez qui on a décelé dès le début de la scolarité des troubles de comportement. Leur étude porte plus précisément sur les services de type psychoé- 
ducatif. La méthodologie a consisté à comparer un groupe test ayant bénéficié d'interventions régulières et un groupe témoin composé d'élèves ne manifestant pas de troubles de comportement. Les résultats obtenus ne manqueront pas de susciter quelques interrogations auprès des chercheurs et des professionnels. Il apparaît en effet que les troubles de comportement demeurent relativement stables malgré les services reçus. Ceux-ci semblent avoir davantage un impact positif sur l'adaptation sociale que sur le rendement scolaire des élèves concernés. Les chercheurs se questionnent dès lors sur la capacité de l'école à assumer une réelle réadaptation des enfants souffrant de troubles graves.

Couture et Lapalme, de leur côté, suivant une méthodologie en grande partie similaire à celle des auteurs de l'article précédemment présenté et basée sur la comparaison entre un groupe test et un groupe témoin, ont cherché à documenter les effets de la mise en place dans deux écoles primaire, de la classe «Kangourou», un modèle appliqué en Angleterre depuis une trentaine d'années et fortement inspiré par la théorie de l'attachement de John Bowlby. L'originalité de cette démarche est de ne pas privilégier une approche strictement individuelle et de repenser la logique de l'intégration des élèves en difficulté dans la classe ordinaire. L'efficacité du modèle est évaluée à partir des perceptions des parents et des enseignants. Ces deux catégories de personnes entourant l'enfant ont une perception très positive de l'impact du fonctionnement de la classe «Kangourou» sur le fonctionnement des enfants. Même si l'étude porte sur une période d'expérimentation limitée, les auteurs estiment que les résultats obtenus permettent de considérer le modèle particulier qu'elles ont étudié comme une ressource prometteuse afin de favoriser la réussite scolaire des jeunes élèves en difficulté de comportement.

Ce numéro fait état de recherches s'intéressant aux pratiques des intervenantes et des intervenants et, dans la majorité des cas, aux effets de celles-ci. Il apporte une contribution originale à l'étude des réussites scolaires en dépassant la réalité de la classe et en privilégiant des perspectives d'études multiples, tant cognitives que psychosociales, contribution qui, nous l'espérons, saura inspirer les chercheuses et les chercheurs ainsi que les praticiennes et les praticiens.

\section{Références}

Bowlby, G. (2005). Taux de décrochage provinciaux: tendances et conséquences. Enquête sur la population active. Statistique Canada. Document téléaccessible à l'adresse <http://www.statcan.ca/francais/freepub/81004-XIF/2005004/drop_f.htm>.

Fortin, L., Marcotte, D., Royer, É. et Potvin, P. (2006). Typology of student at risk of dropping out of school : Description by personal, family and school factors. European Journal of Psychology of Education, XXI(4), 363-383.

Gouvernement du Québec (2001). Programme de formation de l'école québécoise. Éducation préscolaire. Enseignement primaire. Version approuvée. Québec: Ministère de l’Éducation.

Haut Conseil de l'Éducation (2007). L'école primaire. Bilan des résultats de l'École. Paris : République française. 